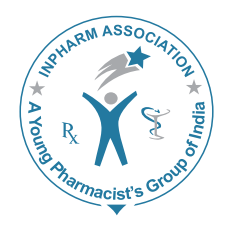

\title{
JIP
}

\section{Design and Microwave-assisted Synthesis of 1,3,4-Oxadiazole Derivatives for Analgesic and Anti-inflammatory Activity}

\author{
Biju CR ${ }^{1,4}$, llango K², Manju Prathap ${ }^{3}$, Rekha K \\ ${ }^{1}$ Department of Pharmaceutical Chemistry, Devaki Amma Memorial College of Pharmacy, Malappuram, \\ Kerala, ${ }^{2}$ SRM College of Pharmacy, SRM University, Kancheepuram, Chennai, Tamil Nadu, ${ }^{3}$ Department of \\ Pharmaceutical Chemistry, Amrutha Institute of Medical Sciences, Cochin, Kerala, ${ }^{4}$ Karpagam University, \\ Echanari, Coimbatore, Tamil Nadu, India
}

Address for correspondence: Mr. Biju CR; E-mail: bijucrmpharm@gmail.com

\begin{abstract}
1,3,4-Oxadizoles form a biologically important group of compounds having activities like analgesic, antiinflammatory, bactericidal, antifungal, anticonvulsant, psychotropic, plant growth regulating and mono amino oxidase inhibition. This research has focused on the incorporation of the oxadiazole moiety into isoniazid because of their versatile biological action, to get 2-aryl-5-(4-pyridyl)-1,3,4-oxadiazole to explore the possibilities of some altered biological action. 1,3,4-Oxadiazole derivatives were synthesized by microwave-assisted synthesis and screened for their analgesic, anti-inflammatory activities. The synthesized compounds were characterized by Melting point, Thin layer chromatographylnfra red, Nuclear magnetic resonance spectroscopy, etc. Almost all the synthesized compounds possessed good activity as compared to the standard.
\end{abstract}

Key words: 1,3,4-Oxadiazole, analgesic, anti-inflammatory, isoniazid

\section{INTRODUCTION}

Microwave-enhanced synthesis ${ }^{[1,2]}$ represents a fundamental step forward in the capabilities of synthetic chemistry. It allows organic chemists to work faster, generating higher yields with increased product purity, and to scale experiments up reliably from milligrams to much larger quantities without the need to alter reaction

\begin{tabular}{|l|l|}
\hline \multicolumn{2}{|c|}{ Access this article online } \\
\hline Quick Response Code: & \\
\hline & Website: \\
\hline & www.jyoungpharm.in \\
& \\
\hline
\end{tabular}

Journal of Young Pharmacists / Vol 4 / No 1 parameters. It offers much more precise control over conditions of temperature and pressure than any previous technology. Ultimately, by eliminating much of the time and effort from the process of performing chemical reactions, it allows chemists to focus on what is most important-the development of new compounds, or refined methods for generating known products. In a solvent-less reaction all the microwave energy is directly absorbed by the reactant molecules. ${ }^{[3]}$ Under these conditions, the non-thermal microwave effect will be operative at high efficiency.

This work aims at the development of a newer isoniazid-based oxadiazole ring system. 1,3,4-Oxadiazole derivatives show a broad spectrum of biological activities, which include analgesic and anti-inflammatory, antimicrobial, anticonvulsant, antifungal, anticancer, 
antimycobacterial, ${ }^{[3,4]}$ etc. The research envisages a meaningful exploration of this lead molecule for novel analgesic, anti-inflammatory activities with minimum toxicity and high potency. ${ }^{[5]}$ The lead compound was structurally modified by incorporating various substitutions at the second and fifth position of the heterocyclic ring system [Table 1]. From a review of the literature it is clear that 2,5 disubstituted 1,3,4-oxadiazole derivatives of oxadiazole possess remarkable analgesic, anti-inflammatory activity. ${ }^{[5,6]}$

\section{MATERIALS AND METHODS}

Microwave-assisted synthetic procedure

Step 1

A mixture of (0.01 mole, $1.37 \mathrm{~g})$ isoniazid, (0.01 mole) aromatic aldehyde and DMF (5 drops) was subjected to microwave irradiation at $300 \mathrm{w}$ internally at $30-\mathrm{sec}$ intervals for $3 \mathrm{~min}$. The reaction mixture was cooled and treated with ice cold water. The resulting solid product was

Table 1: SMI LES and cLog P values of proposed analogues (generated by molinspiration software)

\begin{tabular}{|c|c|c|c|c|}
\hline Compound & $\mathbf{R}_{1}$ & $\mathbf{R}_{2}$ & SMILES notation & $\operatorname{cLog} P$ \\
\hline $2 \mathrm{a}$ & & & COc3cce(c2nnc(c1 cencc1)o2)cc3 & 2.494 \\
\hline $2 b$ & & & COc1 $1 \mathrm{cccc} 1 \mathrm{c} 3 \mathrm{nnc}(\mathrm{c} 2 \mathrm{ccncc} 2) \mathrm{o} 3$ & 2.446 \\
\hline $2 \mathrm{c}$ & & & Oc3ecc(c2nnc(c1 cencc1)o2) $\mathrm{cc} 3$ & 1.958 \\
\hline $2 \mathrm{~d}$ & & $\mathrm{OH}$ & Oc3ecc $(\mathrm{c} 2 \mathrm{nnc}(\mathrm{c} 1 \mathrm{ccncc} 1) \mathrm{o} 2) \mathrm{c}(\mathrm{O}) \mathrm{c} 3$ & 1.667 \\
\hline $2 \mathrm{e}$ & & & $\mathrm{O}=\mathrm{N}(=\mathrm{O}) \mathrm{c} 3 \operatorname{ccc}(\mathrm{c} 2 \mathrm{nnc}(\mathrm{c} 1 \mathrm{ccncc} 1) \mathrm{o} 2) \mathrm{c} 3$ & 2.372 \\
\hline $2 \mathrm{f}$ & & & $\mathrm{O}=\mathrm{N}(=\mathrm{O}) \mathrm{c} 1 \mathrm{ccccc} 1 \mathrm{c} 3 \mathrm{nnc}(\mathrm{c} 2 \mathrm{ccncc} 2) \mathrm{o} 3$ & 2.348 \\
\hline $2 \mathrm{~g}$ & & & $\mathrm{Clc} 3 \mathrm{ccc}(\mathrm{c} 2 \mathrm{nnc}(\mathrm{c} 1 \mathrm{ccncc} 1) \mathrm{o} 2) \mathrm{cc} 3$ & 3.115 \\
\hline $2 \mathrm{~h}$ & & & $\mathrm{Clc} 3 \mathrm{ccc}(\mathrm{c} 2 \mathrm{nnc}(\mathrm{c} 1 \mathrm{ccncc} 1) \mathrm{o} 2) \mathrm{c}(\mathrm{Cl}) \mathrm{c} 3$ & 3.721 \\
\hline $2 \mathrm{i}$ & & & $\mathrm{COc} 3 \mathrm{cc}(\mathrm{c} 2 \mathrm{nnn}(\mathrm{c} 1 \mathrm{ccncc} 1) \mathrm{o} 2) \mathrm{cc}(\mathrm{OC}) \mathrm{c} 3 \mathrm{O}$ & 1.792 \\
\hline
\end{tabular}


filtered, washed with water and recrystallized from ethanol [Table 2] $\cdot^{[7-11]}$

Step 2

To a solution of compound $1 \mathrm{a}$ ( 0.01 mole) in ethanol $(15 \mathrm{ml})$, chloramine- $\mathrm{T}$ ( 0.01 mole) was added. The reaction mixture was exposed to microwave irradiation at $300 \mathrm{~W}$ internally at 30-sec intervals for $4 \mathrm{~min}$. The reaction mixture was cooled and digested with cold water. The solid thus obtained was filtered, washed with water and recrystallized from methanol to give the product [Figure 1]. $\cdot^{[9,11-13]}$

\section{RESULTS AND DISCUSSION}

The purity of the synthesized molecules was ascertained routinely by TLC, and melting points were noted with an open capillary tube method and are uncorrected. ${ }^{[12-16]}$

\section{Infra-red spectral analysis}

Infra-red (IR) spectra were recorded using $\mathrm{KBr}$ pellets in the range of $4000-500 \mathrm{~cm}^{-1}$ on Jasco FTIR model 4100 type A to elucidate the structure of the compounds [Table 3].

${ }^{1} \mathrm{H}$ NMR spectral analysis

Proton NMR (300 MHz) spectra were recorded in $\mathrm{CDCl}_{3}$. Chemical shifts were recorded in parts per million downfield with reference to internal standard Tetra Methyl Silane (TMS) on BurkerAvance DPX 300. The total number of proton obtained from NMR spectra was in accordance with that of respective analogues.

\section{PHARMACOLOGICAL SCREENING}

Acute toxicity study

A prototype molecule was randomly selected for the study of the safety dose range of the analogues. ${ }^{[11,14,15]}$

Table 2: Characteristic ${ }^{1} \mathrm{H}$ NMR spectrum of the synthesized compounds

\begin{tabular}{ll}
\hline Compound & ${ }^{1} \mathrm{H} \mathrm{NMR}\left(\mathrm{CDCl}_{3}\right) \delta \mathrm{ppm}$ \\
\hline $2 \mathrm{a}$ & ${ }^{1} \mathrm{HNMR}\left(\mathrm{CDCl}_{3}\right) \delta \mathrm{ppm}: 2.54(1 \mathrm{H}, \mathrm{O}-\mathrm{H}), 6.13-7.78(\mathrm{Ar}-$ \\
& $\mathrm{H}, 8 \mathrm{H})$ \\
$2 \mathrm{~b}$ & ${ }^{1} \mathrm{HNMR}\left(\mathrm{CDCl}_{3}\right) \delta \mathrm{ppm}: 2.52(1 \mathrm{H}, \mathrm{O}-\mathrm{H}), 4.23-7.9(\mathrm{Ar}-\mathrm{H}$, \\
& $8 \mathrm{H})$ \\
$2 \mathrm{c}$ & ${ }^{1} \mathrm{HNMR}\left(\mathrm{CDCl}_{3}\right) \delta \mathrm{ppm}: 2.40(1 \mathrm{H}, \mathrm{O}-\mathrm{H}), 7.23-7.78(\mathrm{Ar}-$ \\
& $\mathrm{H}, 8 \mathrm{H})$ \\
$2 \mathrm{~d}$ & ${ }^{1} \mathrm{HNMR}\left(\mathrm{CDCl}_{3}\right) \delta \mathrm{ppm}: 2.40(2 \mathrm{H}, \mathrm{O}-\mathrm{H}), 6.22-7.78(\mathrm{Ar}-$ \\
& $\mathrm{H}, 8 \mathrm{H})$ \\
$2 \mathrm{e}$ & ${ }^{1} \mathrm{HNMR}\left(\mathrm{CDCl}_{3}\right) \delta \mathrm{ppm}: 2.85(2 \mathrm{H}, \mathrm{O}-\mathrm{H}), 7.23-6.98(\mathrm{Ar}-$ \\
& $\mathrm{H}, 8 \mathrm{H})$ \\
$2 \mathrm{f}$ & ${ }^{1} \mathrm{HNMR}\left(\mathrm{CDCl}_{3}\right) \delta \mathrm{ppm}: 2.40(1 \mathrm{H}, \mathrm{O}-\mathrm{H}), 8.23-7.88(\mathrm{Ar}-$ \\
& $\mathrm{H}, 8 \mathrm{H})$ \\
\hline
\end{tabular}

Table 3: Characteristic IR peaks of the synthesized compounds

\begin{tabular}{|c|c|}
\hline Compound & IR $\left(\mathrm{KBr} \mathrm{vem}^{-1}\right)$ \\
\hline $2 \mathrm{a}$ & $\begin{array}{l}\text { 3240.79(Methyl C-H stretching),1597.739(C-H bend, } \\
\text { alkyl),1326.79(C-N(stretching(ring)),1151.29 } \\
\text { (Phenolic C-Ostretch),1085.73(Symmetric C-O-C ring } \\
\text { stretch),670.14(aromatic bend) }\end{array}$ \\
\hline $2 b$ & $\begin{array}{l}\text { 3340.79(N-H stretching), } 1597.739 \text { (C-H bend, } \\
\text { alkyl),1326.79(C-N stretching(ring)), } 1151.29 \\
\text { (Phenolic C-Ostretch),1085.73(Symmetric C-O-C ring } \\
\text { stretch),670.14(aromatic bend) }\end{array}$ \\
\hline $2 \mathrm{c}$ & $\begin{array}{l}3322.39(\mathrm{OH}(\text { Phenolic)stretching) }, 1573.63(\mathrm{C}=\mathrm{C} \\
\text { (aromatic)stretching), } 1495.53 \text { (OHbending), } 1325.82 \\
\text { (CN(stretching(ring)),1172.51(asymmetric C-O-C ring } \\
\text { stretch)670.14(C-H aromatic bend) }\end{array}$ \\
\hline $2 \mathrm{~d}$ & $\begin{array}{l}3322.39(\mathrm{OH}(\text { Phenolic) }) \text { tretching }), 1573.63(\mathrm{C}=\mathrm{C} \\
\text { (aromatic)stretching), } 1495.53(\text { OHbending), } 1325.82 \\
\text { (CN(stretching(ring)),1172.51(asymmetric C-O-C ring } \\
\text { stretch)670.14(C-H aromatic bend) }\end{array}$ \\
\hline $2 \mathrm{e}$ & $\begin{array}{l}\text { 3434.6(AromaticCHstretch), } 1529.27 \\
\left.\left(\text { asymmetric }(\mathrm{ArNO})_{2}\right)(\mathrm{N}=\mathrm{O}) \text { stretch }\right), 1411.64(\mathrm{C} \\
\text { Nstretching(ring)),1299.79(symmetric }\left(\mathrm{ArNO}_{2}\right) \\
(\mathrm{N}=\mathrm{O}) \text { stretch }), 1155.15(\text { asymmetric } \mathrm{C}-\mathrm{O}-\mathrm{C} \text { ring } \\
\text { stretch }) 814.77\left(\mathrm{C}-\mathrm{N} \text { stretch }\left(\mathrm{ArNO}_{2}\right)\right)\end{array}$ \\
\hline $2 \mathrm{f}$ & $\begin{array}{l}3434.60(\text { AromaticCHstretch), } 1303.64 \text { (symmetric } \\
\left(\mathrm{ArNO}_{2}\right)(\mathrm{N}=\mathrm{O}) \text { stretch),1159.01(asymmetric C-O-C } \\
\text { ring stretch) }\end{array}$ \\
\hline $2 \mathrm{~g}$ & $\begin{array}{l}3019,(\mathrm{C}-\mathrm{H} \text { str), } 1590.02,(\mathrm{C}=\mathrm{N} \text { imine } \\
\text { stretching }) 1260(\mathrm{C}-\mathrm{O} \text { str}), 820(\mathrm{C}-\text { Haromatic } \\
\text { bending),614.21(C-Cl stretching) }\end{array}$ \\
\hline $2 \mathrm{~h}$ & $\begin{array}{l}3019,(\mathrm{C}-\mathrm{H} \text { str)}, 1590.02,(\mathrm{C}=\mathrm{N} \text { imine } \\
\text { stretching }), 1260(\mathrm{C}-\mathrm{O} \text { str), } 820(\mathrm{C}-\text { Haromatic } \\
\text { bending }), 614.21 \text { (C-Cl stretching) }\end{array}$ \\
\hline $2 \mathrm{i}$ & $\begin{array}{l}3359.39 \text { (O-H stretching), } 3261.04 \text { (Methyl C-H } \\
\text { stretch), } 1495.53 \text { (O-H bending), } 1389.46 \text { (Alkyl } \\
\text { C-H bend), } 1159.01 \text { (Phenolic C-O stretch), } 1097.3 \\
\text { (Symmetric C-O-C (ring) stretch).669.17(C-H bend) }\end{array}$ \\
\hline
\end{tabular}

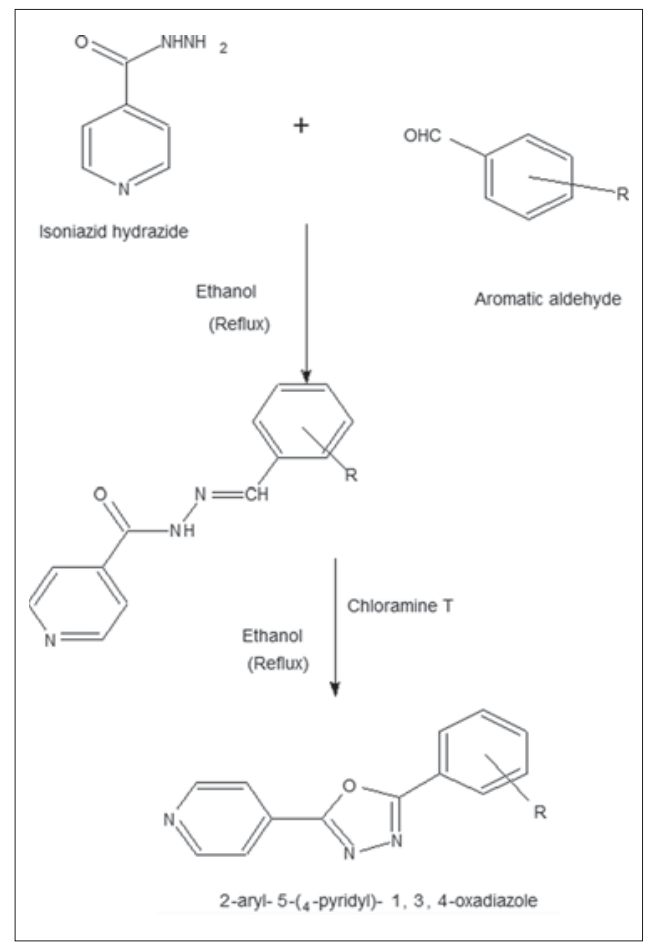

Figure 1: Synthetic scheme of 1,3,4 oxadiazole derivative 
Biju, et al.: Microwave-assisted synthesis of 1,3,4-oxadiazole for anaigesic and anti-inflammatory activity

Table 4: Physicochemical properties of the proposed analogues (generated by ACDLABS software)

\begin{tabular}{lcccc}
\hline Compound & $\begin{array}{c}\text { Molar } \\
\text { refractivity } \mathbf{~ m}^{3}\end{array}$ & Molar volume $\mathbf{~ c m}^{3}$ & Parachor $\mathbf{~ c m}^{3}$ & Polarizability $\mathbf{c m}^{3}$ \\
\hline 2a & $68.68 \pm 0.3$ & $206.4 \pm 3.0$ & $547.0 \pm 4.0$ & $27.23 \pm 0.510^{-24}$ \\
2b & $68.68 \pm 0.3$ & $206.4 \pm 3.0$ & $547.0 \pm 4.0$ & $27.23 \pm 0.510^{-24}$ \\
2c & $63.89 \pm 0.3$ & $180.8 \pm 3.0$ & $505.4 \pm 4.0$ & $25.32 \pm 0.510^{-24}$ \\
2d & $65.77 \pm 0.3$ & $179.3 \pm 3.0$ & $520.4 \pm 4.0$ & $26.07 \pm 0.510^{-24}$ \\
2e & $68.55 \pm 0.3$ & $194.3 \pm 3.0$ & $545.8 \pm 4.0$ & $27.17 \pm 0.510^{-24}$ \\
2f & $68.55 \pm 0.3$ & $194.3 \pm 3.0$ & $545.8 \pm 4.0$ & $27.17 \pm 0.510^{-24}$ \\
2g & $66.90 \pm 0.3$ & $194.4 \pm 3.0$ & $526.2 \pm 4.0$ & 2.958 \\
2h & $71.80 \pm 0.3$ & $206.3 \pm 3.0$ & $562.1 \pm 4.0$ & 2.372 \\
2i & $77.24 \pm 0.3$ & $228.9 \pm 3.0$ & $=618.7 \pm 4.0$ & $2.3 .46 \pm 0.510^{-24}$ \\
\hline
\end{tabular}

Table 5: Physicochemical data of newly synthesized compounds

\begin{tabular}{llcccc}
\hline Compound & Substituent & $\begin{array}{c}\text { Molecular } \\
\text { formula }\end{array}$ & $\begin{array}{c}\text { Molecular } \\
\text { weight }\end{array}$ & $\mathbf{m p}^{\circ} \mathrm{C}$ & $\mathbf{R}_{\mathrm{f}}$ \\
\hline $\mathrm{C}_{14} \mathrm{H}_{11} \mathrm{~N}_{3} \mathrm{O}_{2}$ & 253.261 & 163 & 0.57 \\
& & & & &
\end{tabular}

$2 b$<smiles>COc1ccccc1C</smiles>

2c<smiles>Cc1ccc(O)cc1</smiles>

$2 d$<smiles>Cc1ccc(O)cc1O</smiles>

$2 \mathrm{e}$<smiles>Cc1cccc([N+](=O)[O-])c1</smiles>

$2 \mathrm{f}$<smiles>Cc1ccccc1[N+](=O)[O-]</smiles>

$2 \mathrm{~g}$<smiles>Cc1ccc(Cl)cc1</smiles>

$2 \mathrm{~h}$<smiles>Cc1ccc(Cl)cc1Cl</smiles>

$2 \mathrm{i}$

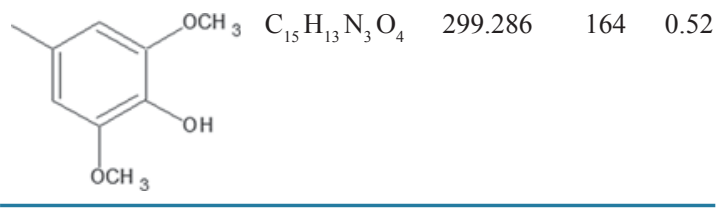

In this study, it was found that up to $1600 \mathrm{mg} / \mathrm{kg}$ dose, the compound is safe. i.e. there was no mortality or gross
Table 6: Analgesic activity (acetic acid-induced Writhing method)

\begin{tabular}{lccc}
\hline Name of group & Treatment & $\begin{array}{c}\text { No. of Writhing } \\
\text { in 20 min } \\
\text { (mean+SEM) }\end{array}$ & $\begin{array}{c}\text { Percentage } \\
\text { reduction of } \\
\text { Writhing }\end{array}$ \\
\hline Vehicle control (1\%CMC) & $20 \mathrm{mg} / \mathrm{kg}$ & $39.2 \pm 0.04$ & - \\
Aspirin & $40 \mathrm{mg} / \mathrm{kg}$ & $16.4 \pm 0.08$ & 58.16 \\
$2 \mathrm{a}$ & $500 \mathrm{mg} / \mathrm{kg}$ & $13.6 \pm 0.74$ & 65.30 \\
$2 \mathrm{c}$ & $500 \mathrm{mg} / \mathrm{kg}$ & $12.8 \pm 0.48$ & 67.34 \\
$2 \mathrm{e}$ & $500 \mathrm{mg} / \mathrm{kg}$ & $19.9 \pm 0.74$ & 49.23 \\
$2 \mathrm{~g}$ & $500 \mathrm{mg} / \mathrm{kg}$ & $19.4 \pm 0.87$ & 50.51 \\
$2 \mathrm{l}$ & $500 \mathrm{mg} / \mathrm{kg}$ & $16.2 \pm 0.73$ & 58.86 \\
\hline
\end{tabular}

Table 7: Anti-inflammatory activity (Carageenaninduced rat paw edema method)

\begin{tabular}{lccc}
\hline Treatment & Dose (per Kg) & $\begin{array}{c}\text { Mean difference } \\
\text { in paw } \\
\text { thickness }=\text { SEM }\end{array}$ & $\begin{array}{c}\text { Percentage } \\
\text { inhibition } \\
\text { of edema }\end{array}$ \\
\hline Vehicle control (1\%CMC) & $20 \mathrm{mg} / \mathrm{kg}$ & $2.23 \pm 0.09$ & - \\
Indomethacin & $20 \mathrm{mg} / \mathrm{kg}$ & $0.72 \pm 0.08$ & 67.71 \\
$2 \mathrm{a}$ & $500 \mathrm{mg} / \mathrm{kg}$ & $0.70 \pm 0.03$ & 68.60 \\
$2 \mathrm{c}$ & $500 \mathrm{mg} / \mathrm{kg}$ & $0.69 \pm 0.04$ & 69.05 \\
$2 \mathrm{e}$ & $500 \mathrm{mg} / \mathrm{kg}$ & $0.96 \pm 0.03$ & 56.95 \\
$2 \mathrm{~g}$ & $500 \mathrm{mg} / \mathrm{kg}$ & $0.89 \pm 0.02$ & 60.08 \\
21 & $500 \mathrm{mg} / \mathrm{kg}$ & $0.86 \pm 0.04$ & 61.43 \\
\hline
\end{tabular}

behavioral change in the animals used. ${ }^{[17-24]}$

\section{SUMMARY AND CONCLUSION}

This research work was focused on the rational approach in the design and development of 1,3,4 oxadiazole derivatives as novel analgesic, anti-inflammatory drugs.

The candidates which obeyed the Lipinski rule of five were taken for wet lab synthesis. Nine different analogues were synthesized by microwave methods and the purity of the compounds thus synthesized was ascertained by consistency in melting point and $\mathrm{Rf}$ value and characterized by UV, IR and ${ }^{1} \mathrm{H}$ NMR spectral studies [Tables 4 and 5].

Among the newly synthesized 1,3,4 oxadiazole analogues 
five were screened for analgesic and anti-inflammatory activity and the compounds $2 \mathrm{a}, 2 \mathrm{c}$ and $2 \mathrm{i}$ showed good analgesic and anti-inflammatory activity. Acute toxicity studies showed that the analogues were safe with low toxicity. So these derivatives may be future leads for analgesic and anti-inflammatory drug discovery [Tables 6 and 7].

\section{REFERENCES}

1. Khan MS, Chawla G, Mueed MA. Synthesis characterization and biological evaluation of substituted oxadiazole, and triazole derivatives. Indian J Chem 2004;43B:1302-5.

2. Zheng L, Wang X, China PR, Wang X. Synthesis and antibacterial activities of 1,3,4-oxadiazole derivatives. Indian J Chem 2003;42B:941.

3. Govt. of India, Ministry of Health and Family Wefare, Indian Pharmacopoeia. Delhi: Controller of Publications; 1996. p. 408.

4. Khan MS, Anther M. Microwave assisted synthesis of 2,5- disubstituted 1,3,4- oxadiazole derivatives. Indian J Chem 2003;42B:900-4.

5. Hui XP, CnuCH, Zhang ZY. Synthesis and anti -inflammatory activities of 1,3,4-oxadiazole derivatives containing 5-methylisoxazole moiety. Indian J Chem 2002;41B:2176-9.

6. David AW, Thomas LL. Foye's Principles of medicinal chemistry. $5^{\text {th }}$ ed. New York: Lippincott Williams and Wilkins; 2002. p. 12-7.

7. King FB. Medicinal chemistry Principles and practice. $2^{\text {nd }}$ ed. UK: Royal Society of Chemistry; 2002.

8. Thomas G. Fundamentals of medicinal chemistry. New York: John Wiley and Sons; 2003. p. 57-61.

9. Manfred EW. Burger's medicinal chemistry and drug discovery. $5^{\text {th }}$ ed. New York: John Wiley and Sons; Synthesis and anti-inflammatory activity of 1,3,4-oxadiazole derivatives 2003. p. 10-6.

10. Patrick GL. An introduction to medicinal chemistry. $2^{\text {nd }}$ ed. New York: Oxford University Press; 2001. 142-7.

11. Rand HP, Dale MM, Ritter MM, Moore PK. Pharmacology. $5^{\text {th }}$ ed. NewYork: Churchill Livingstone; 2003. p. 51-63.
12. David AW, Thomas LL.Foye's Principles of medicinal chemistry. $5^{\text {th }}$ ed. New York: Lippincott Williams and Wilkins; 2002. p. 751-94.

13. Jaime ND, William AR. Wilson and Gisvold's text book of organic medicinal and pharmaceutical chemistry. $10^{\text {th }}$ ed. New York: Lippincott-Raven; 1998. p. 687-795.

14. Goodman and Gilman's, The Pharmacological basis of therapeutics. $11^{\text {th }}$ ed.1998. Mc Graw Hill company Publication, New york. p. 617-47.

15. JaimeND, William AR. Wilson and Gisvold's text book of organic medicinal and pharmaceutical chemistry. $11^{\text {th }}$ ed. New York: Lippincott-Raven; 2004. p. 822-9.

16. Chemical and Engineering News, American Chemical Soc. Vol. 80. 2002. p. 16.

17. Patrick GL. An introduction to medicinal chemistry. $2^{\text {nd }} \mathrm{ed}$. New York: Oxford University Press; 2001. p. 258-88.

18. Kappe CO, Dallinger D. The impact of microwave synthesis on drug discovery. Nat Rev Drug Discov 2006;5:51-63.

19. Abd el-Samii ZK.Synthesis and anti-inflammatory activity of some novel 1,3,4-oxadiazole derivatives. J Chem Technol Biotechnol 1992;53:143-6.

20. Mullican MD, Wilson MW, Connor DT, Kostlan CR, Schrier DJ, Dyer RD. Design of 5-(3,5-di-tert-butyl-4-hydroxyphenyl)-1,3,4thiadiazoles, -1,3,4-oxadiazoles, and -1,2,4-triazoles as orally-active, nonulcerogenicantiinflammatory agents. J Med Chem 1993;36:1090-9.

21. Boschelli DH, Connor DT, Bornemeier DA, Dyer RD, Kennedy JA, Kuipers PJ, et al. 1,3,4-Oxadiazole, 1,3,4-thiadiazole, and 1,2,4-triazole analogs of the fenamates: In vitro inhibition of cyclooxygenase and 5-lipoxygenase activities. J Med Chem 1993;36:1802-10.

22. Omar FA. Synthesis of some novel 1,3,4-oxadiazole derivatives for anti diabetic activity. EurJ Med Chem 1996;31:819-25.

23. Palaska E, Sahin G, Kelicen P, Durlu NT, Altinok G. Synthesis and antiinflammatory activity of 1-acylthiosemicarbazides, 1,3,4-oxadiazoles, 1,3,4-thiadiazoles and 1,2,4-triazole-3-thiones. Farmaco 2002;57:101-7.

24. Jakubkiene V, Burbuliene MM, Mekuskiene G, Udrenaite E, Gaidelis P, Vainilavicius P. Synthesis and anti-inflammatory activity of 5-(6-methyl-2substituted 4-pyrimidinyloxymethyl)-1,3,4-oxadiazole-2-thiones and their 3-morpholinomethyl derivatives. Farmaco 2003;58:323-8.

How to cite this article: Biju CR, llango K, Prathap M, Rekha K. Design and microwave-assisted synthesis of 1,3,4-oxadiazole derivatives for analgesic and anti-inflammatory activity. J Young Pharmacists 2012;4:33-7.

Source of Support: Nil, Conflict of Interest: None declared.

\section{Staying in touch with the journal}

1) Table of Contents (TOC) email alert

Receive an email alert containing the TOC when a new complete issue of the journal is made available online. To register for TOC alerts go to http://www.jyoungpharm.in/signup.asp.

\section{2) RSS feeds}

Really Simple Syndication (RSS) helps you to get alerts on new publication right on your desktop without going to the journal's website. You need a software (e.g. RSSReader, Feed Demon, FeedReader, My Yahoo!, NewsGator and NewzCrawler) to get advantage of this tool. RSS feeds can also be read through FireFox or Microsoft Outlook 2007. Once any of these small (and mostly free) software is installed, add http://www.jyoungpharm.in/rssfeed.asp as one of the feeds. 